Abstracta Iranica

Revue bibliographique pour le domaine irano-aryen

Volume 40-41 | 2019

Comptes rendus des publications de 2017-2018

\title{
Assadullah Souren Melikian-Chirvani (ed). The World of the Fatimids
}

\section{Frantz Chaigne}

\section{(2) OpenEdition \\ 12 Journals}

\section{Édition électronique}

URL : http://journals.openedition.org/abstractairanica/51411

DOI : 10.4000/abstractairanica.51411

ISBN : 1961-960X

ISSN : 1961-960X

Éditeur :

CNRS (UMR 7528 Mondes iraniens et indiens), Éditions de l'IFRI

Référence électronique

Frantz Chaigne, "Assadullah Souren Melikian-Chirvani (ed). The World of the Fatimids », Abstracta

Iranica [En ligne], Volume 40-41 | 2019, document 24, mis en ligne le 30 décembre 2019, consulté le 17 avril 2021. URL : http://journals.openedition.org/abstractairanica/51411 ; DOI : https://doi.org/ 10.4000/abstractairanica. 51411

Ce document a été généré automatiquement le 17 avril 2021.

Tous droits réservés 


\title{
Assadullah Souren Melikian- Chirvani (ed). The World of the Fatimids
}

\author{
Frantz Chaigne
}

\section{RÉFÉRENCE}

Assadullah Souren Melikian-Chirvani (ed). The World of the Fatimids. Munich: Hirmer, 2018, 376 p., ISBN: 9783777430379

1 Le présent catalogue a été publié à l'occasion d'une exposition qui s'est tenue en 2018 à l'Aga Khan Museum de Toronto. Il mérite d'être ici recensé dans la mesure où les interactions entre les mondes persan et fatimide y sont largement abordées. Il comprend quatorze essais, une bibliographie, des notes sur les pièces exposées (malheureusement sans numéros de catalogue), ainsi que des index historique, géographique et par typologies d'objets.

2 Le chapitre 3, «Fatimid Art and Its Unresolved Enigmas: From Ceramics to Rock Crystal to Its Vanished Silverware» (A. S. Melikian-Chirvani), est le plus long de l'ouvrage. Nous ne l'aborderons ici pour chaque support qu'au prisme des échanges avec la sphère iranienne. Concernant la céramique, l'auteur y esquisse une démonstration qu'il poursuivra au chapitre 14 quant à la nature des pièces décrites par Naser-e Khosrow dans son Safar-Name et sur laquelle nous reviendrons. Il soulève aussi le problème de l'importation de la technique du lustre métallique en Égypte et montre que des considérations politiques et aussi esthétiques incitent à ne pas privilégier Bagdad au détriment de l'Iran. Si l'iconographie relève le plus souvent d'une esthétique propre à la culture fatimide, certaines pièces - et non des moindres - regardent objectivement vers les canons sassanides, à l'instar de celles portant la signature «Muslim ». Concernant les artefacts en cristal de roche, l'auteur part là-aussi des sources et cite Jowhari Nishaburi qui atteste de leur présence en Iran, sans doute suite à l'exil de 
princes consécutivement à la chute de la dynastie. Melikian-Chirvani souligne aussi la nécessité de continuer à questionner ces pièces, à l'instar d'A. Shalem qui a pu montrer que certaines d'entre elles devraient plutôt être réattribuées à l'Iran oriental au vu de leurs motifs. L'examen de certaines œuvres en métal, en bois et en ivoire atteste là encore de connexions, sinon de transferts depuis l'Iran.

Les chapitres 13, "The Iranian Da'is and Fatimid Egypt " (F. Daftary), et 14, «Just What Did Naser-e Khosrow See in Cairo? An Ongoing Debate» (A.S. Melikian-Chirvani) s'inscrivent quant à eux dans une partie dévolue à la place des Fatimides sur la scène internationale. Le premier de ces deux essais relève uniquement de problématiques religieuses et historiques et ne sera donc pas commenté ici. Dans le chapitre 14, A. S. Melikian-Chirvani approfondit des questions esquissées dans le chapitre 3. Dans un premier temps, il se livre à des analyses terminologiques lui permettant de contrer les assertions d'O. Watson quant à l'identification des céramiques décrites par Naser-e Khosrow comme étant du lustre métallique; selon Melikian-Chirvani, il s'agirait d'artefacts désormais disparus et donc non identifiables mais ne relevant en aucun cas de la technique du lustre. Puis il revient sur les origines des iconographies rencontrées sur les pièces de forme fatimides décorées de lustre métallique, soulignant au passage la difficulté d'évoquer les transferts entre les sphères iranienne et égyptienne. Enfin, il revient sur la difficulté d'interpréter correctement les sources se basant cette fois sur la concision de la description d'un trône par le voyageur persan.

Quelques items d'origine iranienne font l'objet de brèves notices à la fin de l'ouvrage. Il s'agit de deux bols et d'une coupe à libation en céramique, ainsi que d'un flacon et de dix pièces d'échec en cristal de roche.

\section{AUTEURS}

\section{FRANTZ CHAIGNE}

Chercheur associé, CNRS, Orient \& Méditerranée-«Islam médiéval» 